\title{
Small cell neuroendocrine carcinoma: A rare second primary malignancy after treatment of nasopharyngeal carcinoma
}

\section{Krishnamoorthy Madhusudhan ${ }^{1}$, Nor Eyzawiah binti Hassan², Norasnieda binti Md Shukri ${ }^{3}$, Shahrul bin Hitam, Ikmal Hisyam bin Bakrin ${ }^{5}$}

${ }^{1}$ Department of Ear, Nose, Throat and Head \&Neck Surgery, Hospital Ampang, Selangor, Malaysia

${ }^{2}$ Faculty of Medicine and Health Sciences, University Sains Islam Malaysia, Negeri Sembilan, Malaysia

${ }^{3}$ School of Medical Sciences, University Sains Malaysia, Kelantan, Malaysia

${ }^{4}$ Ear, Nose, Throat and Head \&Neck Surgery Department, Hospital Ampang, Selangor, Malaysia

${ }^{5}$ Department of Pathology, University Putra Malaysia, Selangor, Malaysia

\section{ABSTRACT}

Small cell neuroendocrine carcinoma (SCNEC) of the nasopharynx and nasal cavity is a rare condition. It is an aggressive malignancy with a high recurrence rate. Despite its rarity in the sinonasal region, it may occur as a second primary malignancy. Patients with cancer of the head and neck region are more prone to develop a second primary tumor due to the field cancerization phenomenon, and a higher incidence is seen in those who have previously received radiation therapy. A detailed clinical and histopathological examination is pivotal to diagnose SCNEC as a second primary malignancy. We wish to highlight such a peculiar case from our center.

KEYWORDS: neuroendocrine carcinoma, nasopharyngeal carcinoma, second primary malignancy, small cell carcinoma.

\section{INTRODUCTION}

Patients who have successfully received treatment with radiation therapy have a risk of developing a new malignancy later on ${ }^{1}$. An example of such an entity is the development of small cell neuroendocrine carcinoma (SCNEG) after successful treatment of nasopharyngeal carcinoma (NPC) by radiation therapy. Extrapulmonary small cell carcinomas are rare, accounting for only $0.1-0.4 \%$ of all malignancies and 2.5-4\% of all small cell carcinomas $^{2}$. Such a tumor occurring in the sinonasal tract is very uncommon. However, when such a tumor occurs as a second primary malignancy in the nasopharynx, it represents an even rarer situation ${ }^{3}$.

\section{CASE REPORT}

A 68-year-old female presented with bloodstained nasal discharge for two weeks prior to consultation. She had T1N3M0 non-keratinizing squamous cell carcinoma of the nasopharynx 4 years before (confirmatory biopsy was taken from the right fossa of Rosenmüller). She was successfully treated with concurrent chemo- and radio-therapy (CCRT). Radiation therapy was divided into two phases. In the first phase, she received a total dose of 40 Gray (Gy) in 20 fractions to the neck and fasciocervical region. In the second phase, she received a total of $30 \mathrm{~Gy}$ in 15 fractions to the fasciocervical region, then $20 \mathrm{~Gy}$ in 10 fractions to the anterior neck and finally 26 Gy in 13 fractions to the right and left side of the posterior neck. After the radiation therapy, she underwent 5 cycles of chemotherapy (cisplatin) on a weekly basis. The $6^{\text {th }}$ cycle of cisplatin was withheld due to leukopenia. Upon completion of treatment, she was monitored closely and was in remission. During her $4^{\text {th }}$ year of routine surveillance, a rigid nasal endoscopy revealed a suspicious mass over the posterior free margin of the nasal septum (right side) (Figure 1).

Subsequently, a Positron Emission Tomography

Corresponding author: Dr. Madhusudhan Krishnamoorthy (MBBS), ENT Department, Hospital Ampang, Jalan Mewah Utara, Pandan Mewah, 68000 Ampang, Selangor, Malaysia 


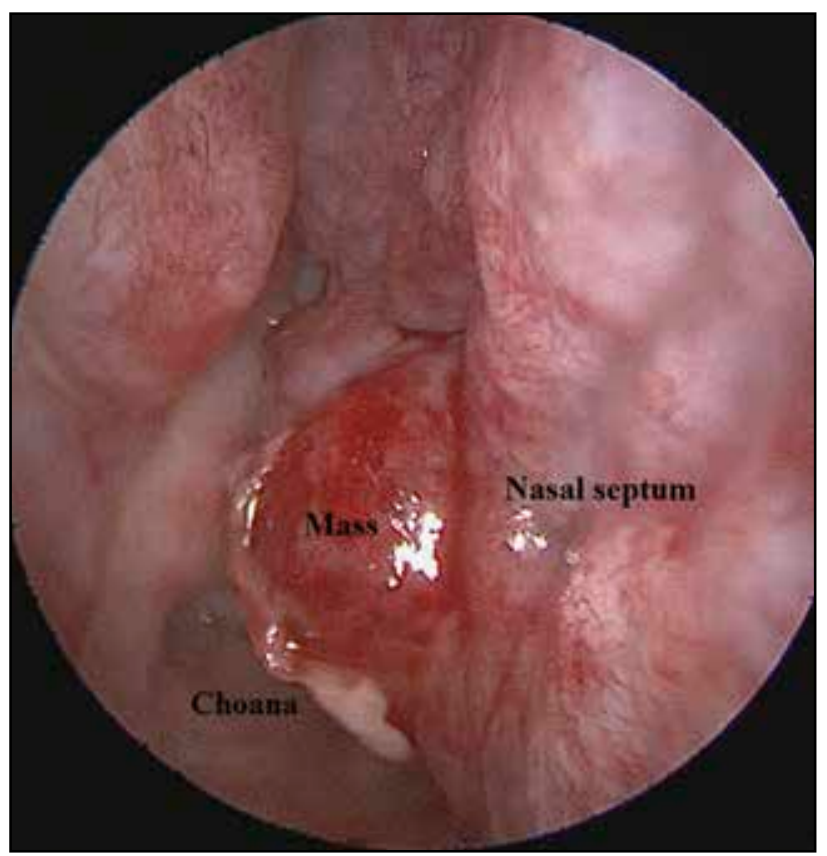

Figure 1 Endoscopic view of the posterior free margin of the nasal septum (right side).

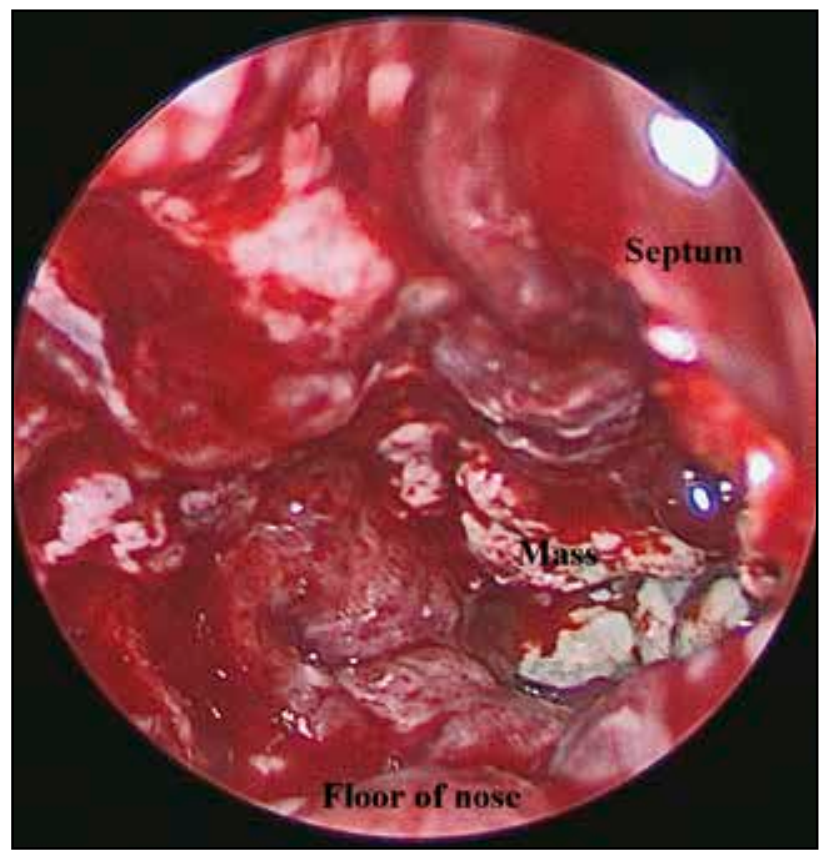

Figure 3 Endoscopic view of the nasopharynx showing a yellowish-white mass obscuring the entire nasopharynx.

- computed tomography (PET-CT) scan was done. This scan confirmed fluorodeoxyglucose $\left({ }^{18} \mathrm{~F}-\mathrm{FDG}\right)$ avid malignancy of the soft tissue mass over the posterior end of the nasal septum's right side with a Standardized Uptake Value (SUV) of 6.2. There was also a bulky posterior nasopharynx (left side) with a SUV of 5.2 (Figure 2).

Otherwise, the nasal cavity and the rest of the

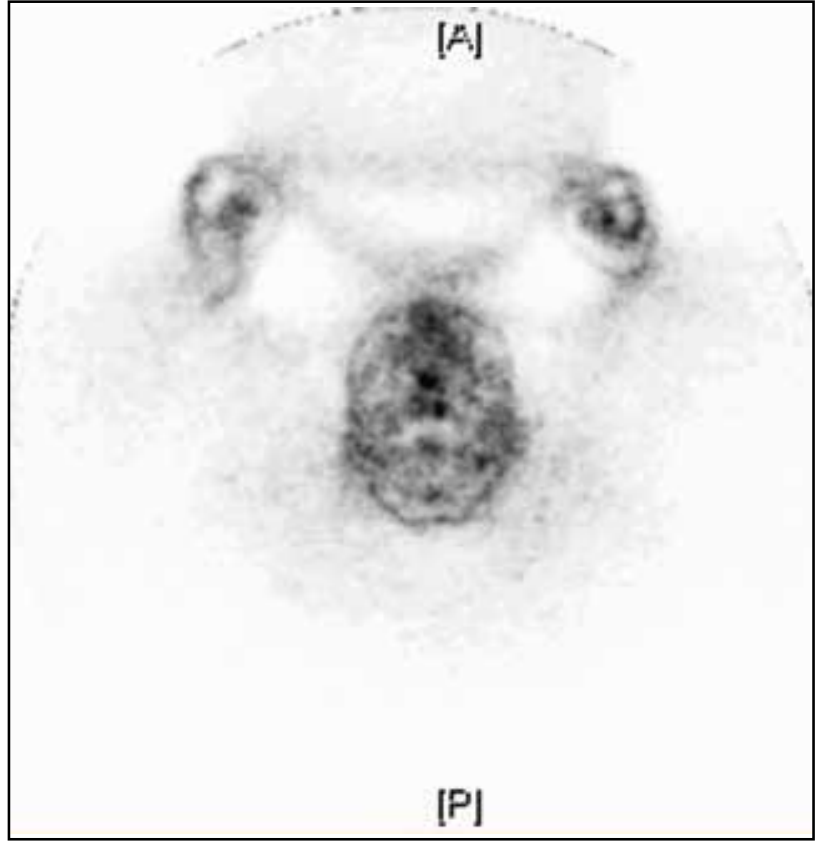

Figure 2 PET-CT scan showing avid malignancy of the soft tissue mass over the posterior end of the nasal septum's right side with Standardized Uptake Value (SUV) of 6.2, as well as a bulky posterior nasopharynx (left side) with a SUV of 5.2.

body were free of disease.

A wide local excision of the nasal mass along with posterior septectomy was done successfully under general anaesthesia. The histologic examination confirmed a diagnosis of neuroendocrine carcinoma. She was scheduled for adjuvant Intensity Modulated Radiotherapy; however, she was undecided for the treatment. She was followed up closely with no evidence of obvious local residue or recurrence of the disease.

In the $4^{\text {th }}$ post-operative month she complained of reduced hearing in her right ear and a severe throbbing headache. A detailed clinical examination revealed a right-sided middle ear effusion with no other anomalies. The rigid nasal endoscopy showed that the nasal cavity mucosa on both sides was severely congested. There was a yellowish-white mass occluding the entire nasopharynx (Figure 3).

Biopsies of the mass taken under general anaesthesia confirmed high-grade SCNEC. The microscopic examination of the lesion using haematoxylin and eosin (H\&E) stain showed multiple fragments of tumor tissue focally covered by respiratory epithelium. The underlying stroma showed malignant cells infiltrating in trabeculae, irregular nests and islands accompanied by prominent vascular channels and a moderate lymphoplasmacytic cell infiltrate (Figure 4). The cells displayed round to oval nuclei with fine stippled chromatin pattern and indistinct scanty cyto- 


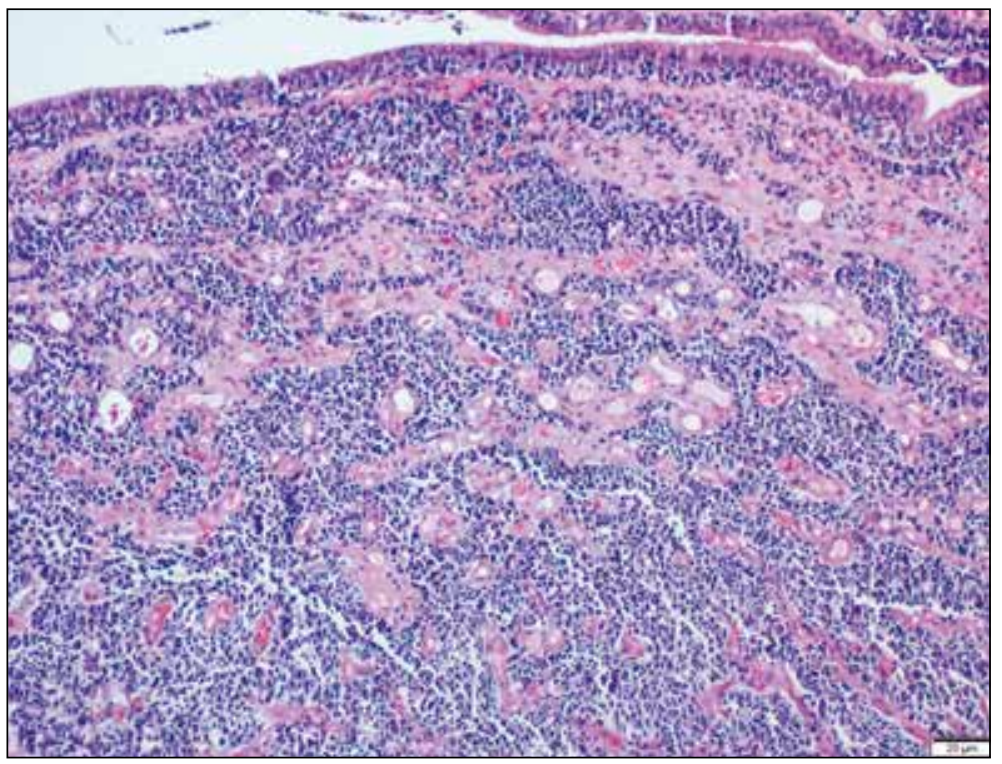

Figure 4 Malignant cells arranged in trabeculae, irregular nests and islands accompanied by prominent vascular channels. The overlying respiratory epithelium was unremarkable (Hematoxylin and eosin stain, original magnification $\mathrm{x} 100$ ).

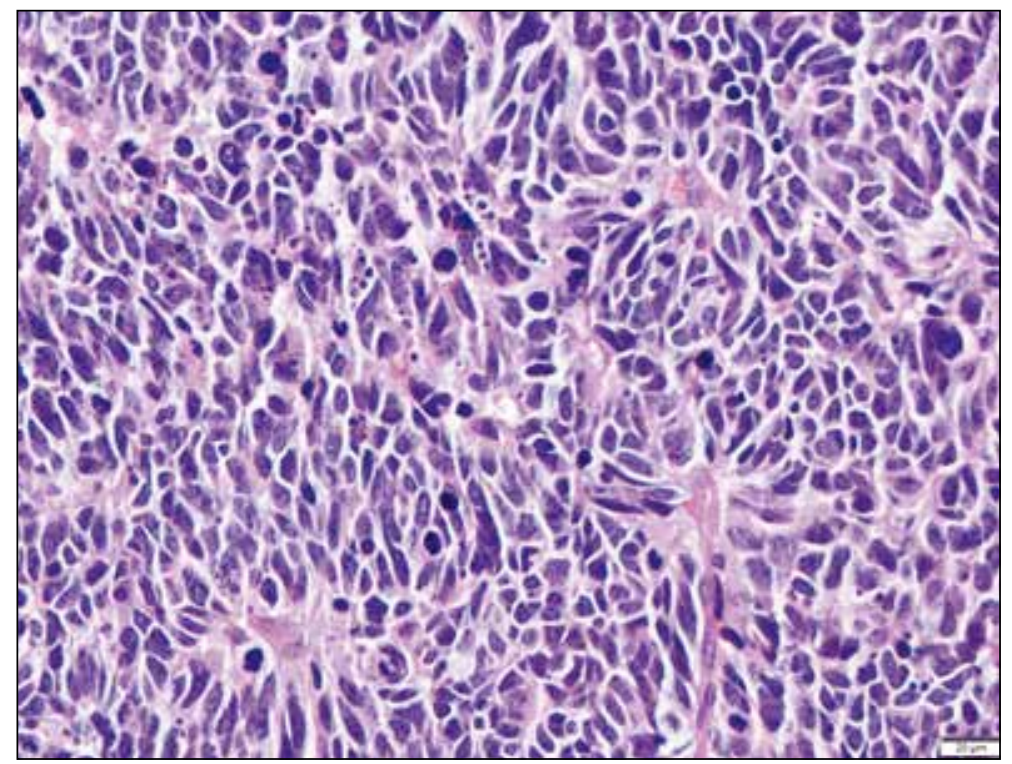

Figure 5 The malignant cells displayed round to oval nuclei with fine stippled chromatin pattern and indistinct scanty cytoplasm. Mitotic figures and apoptotic bodies were frequently seen. Occasional nuclear moulding was also noted (Hematoxylin and eosin stain, original magnification $\times 400$ ).

plasm. Mitotic figures and apoptotic bodies were frequently seen. Occasional nuclear molding and tumor necrosis were also noted (Figure 5). The cells were immunoreactive for CKAE1/AE3 (diffuse, cytoplasmic \& perinuclear dot-like positivity), Synaptophysin (diffuse), and Chromogranin A (in one focus). The Ki-67 proliferative index was $99 \%$. Other markers to rule out differential diagnosis of sinonasal tract tumors were negative
(S100, LCA, CD99, SMA and desmin). There was no squamous or glandular differentiation observed. The overlying respiratory and squamous epithelium was unremarkable. Based on the histological and immunohistochemical profile, a diagnosis of high-grade SCNEC was made.

Staging computed tomography (CT) scan revealed liver metastasis and she succumbed to her disease shortly thereafter. 


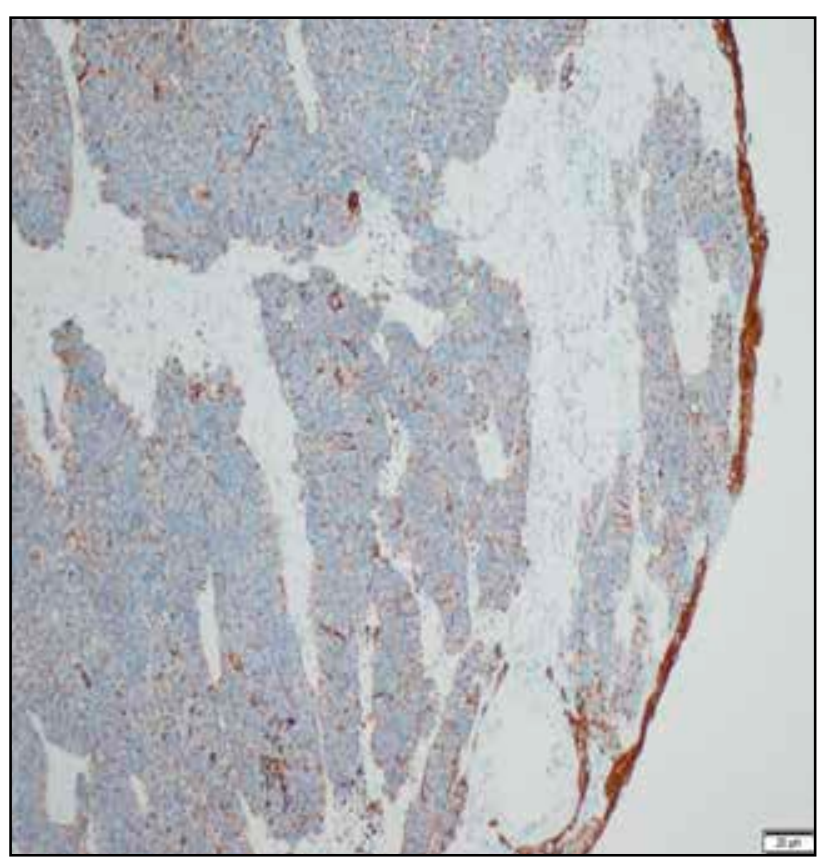

Figure 6 The malignant cells were immunoreactive for CKAE1/AE3 (Immunohistochemical stain, original magnification $\times 100$ ).

\section{DISCUSSIONS}

Sinonasal malignancies make up less than $5 \%$ of all head and neck cancers ${ }^{4}$. Neoplasms of various cellular origin including epithelial (most common $)^{5,6}$, mesenchymal and neuroectodermal differentiation may arise in the sinonasal tract. Malignancies with neuroendocrine differentiation are rare in the sinonasal tract $t^{6}$. A tumor of such differentiation is rarely seen in the head and neck region $^{3}$, the lungs being the commonest primary site $^{7,8}$. Neuroendocrine tumors may arise in sites that typically do not contain neuroendocrine cells. Such sites do not imply embryologic derivation from neuroectoderm. It only reflects a shared phenotype characterized by expression of multiple genes encoding both the neuronal and the endocrine feature ${ }^{9}$.

Extra pulmonary SCNECs are rare, accounting for only $0.1-0.4 \%$ of all malignancies ${ }^{2}$ and $2.5-5 \%$ of all SCNECs ${ }^{7}$, with the larynx being the commonest extrapulmonary site involved ${ }^{8}$. The incidence of a second primary malignancy developing in patients who were previously treated with radiotherapy for nasopharyngeal carcinoma was reported at $0.7 \%$, with onset occurring 5-18 years after the treatment ${ }^{8}$. Much of the cellular and molecular evidence support the field metastasis theory. SCNEC is a highgrade poorly differentiated neuroendocrine tumor ${ }^{7}$ which is known for its cellular aggressiveness and poor prognosis ${ }^{3}$. SCNEC is also known as poorly dif-

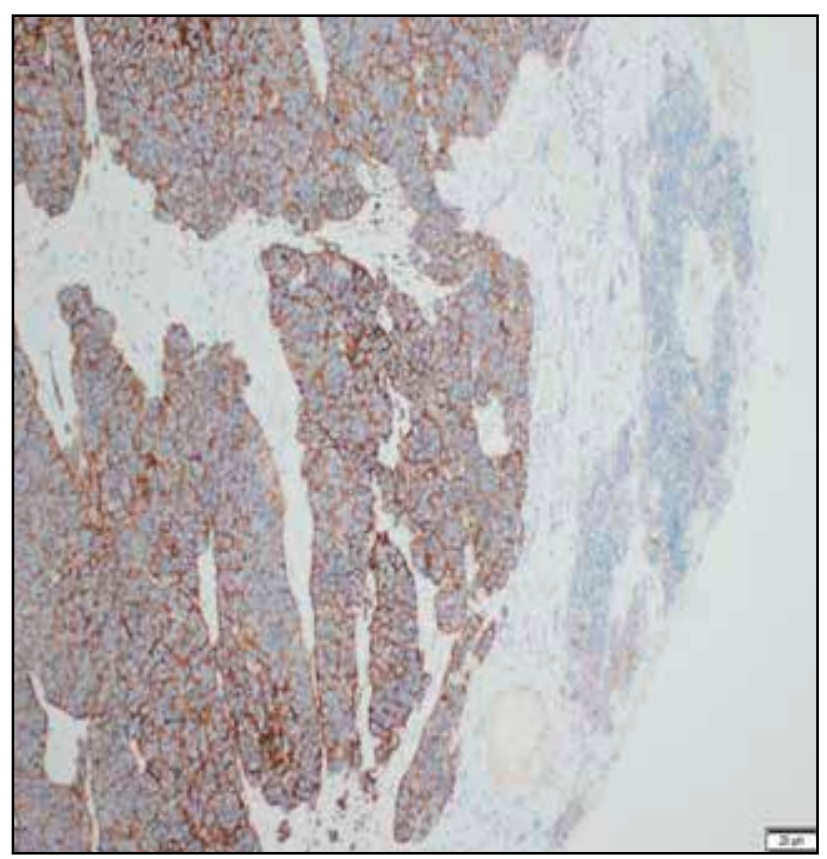

Figure 7 The malignant cells were immunoreactive for Synaptophysin (Immunohistochemical stain, original magnification $\mathrm{x} 100$ ).

ferentiated neuroendocrine carcinoma. Barnard was the first to describe it in 1926, using the term oat cell sarcoma ${ }^{3,8}$. Silva et al. went on to describe sinonasal neuroendocrine carcinoma in $1982^{10}$. Reports show that the median age of presentation of patients with sinonasal neuroendocrine carcinoma ranges between $50-57$ years ${ }^{4}$.

Sinonasal primary neuroendocrine tumors are represented by a spectrum of four major histologic phenotypes: esthesioneuroblastoma (ENB), sinonasal undifferentiated carcinoma (SNUG), neuroendocrine carcinoma and small cell undifferentiated carcinoma ${ }^{11}$. However, due to the rarity of this disease, with no definite classification, the World Health Organization (WHO) addressed this issue in 2005 and classified it as: i) typical carcinoid, ii) atypical carcinoid, iii) small cell carcinoma neuroendocrine type, iv) sinonasal neuroendocrine carcinoma not otherwise specified and v) paraganglioma ${ }^{4}$. SNUC and ENB were not included in the WHO classification.

As evidenced in our case, SCNEC of the nasopharynx may present with a yellowish-white mass ${ }^{5}$. Our patient developed SCNEC as a second primary malignancy 4 years after successful completion of CCRT treatment. To establish a newly diagnosed second primary malignancy in the nasal cavity or nasopharynx, various criteria must be met. Each neoplasm must be geographically disparate with no dysplasia in the intervening mucosa, the possibility of the second primary tumor representing a metas- 


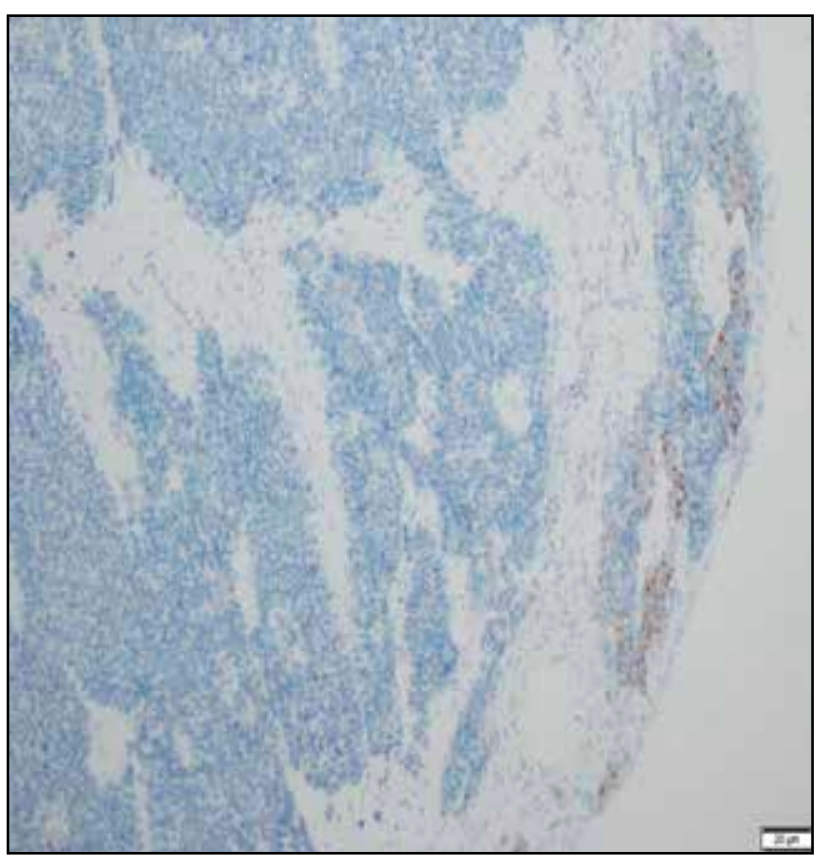

Figure 8 The malignant cells were immunoreactive for Chromogranin A in a focal area (Immunohistochemical stain, original magnification $x$ 100).

tasis or relapse must be excluded, the second primary malignancy has to be separated from the first by at least $2 \mathrm{~cm}$ of normal epithelium, or, has to occur at least 3 years after diagnosing the first primary cancer ${ }^{1}$. The diagnosis of a second primary malignancy in our case is based on the aforementioned criteria. Firstly, the second tumor occurred 4 years after the treatment of NPC. Secondly, during the patient's entire surveillance period post CCRT, there was no evidence of recurrence. Thirdly, the second tumor was localized in the nasal septum and there was only a minimal involvement of the left side of the nasopharynx (based on the PET-CT scan) and, lastly, the histopathologic evidence showed difference of tumor type from the initial one and this second primary malignancy. The patient subsequently developed local recurrence of disease, despite complete surgical excision with clear margins (R0 resection). This is in keeping with the fact that SCNECs are aggressive tumors with a local recurrence rate of $45 \%$.

Conventional microscopic histopathologic examination alone does not suffice to arrive at the diagnosis of SCNEC. Further immunohistochemical studies help to exclude other sinonasal neoplasms such as lymphoma, ENB, malignant melanoma, and $\mathrm{SNUC}^{5}$. Although SCNEC can be quite similar to ENB morphologically and immunohistologically, there are specific features that can be used to differentiate these two mimickers. ENB shows neurofibrillary background, with evi-

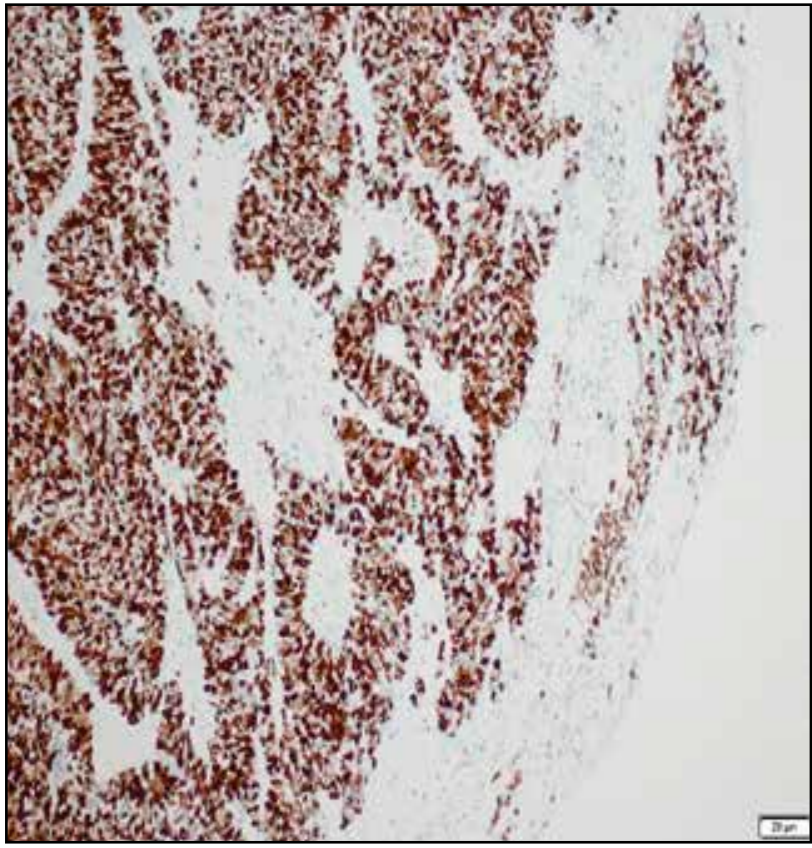

Figure 9 The Ki-67 proliferative index was high, 99\% (Immunohistochemical stain, original magnification $\times 100$ ).

dence of Homer Wright pseudorosette formations ${ }^{8}$. Furthermore, ENB shows immunoreactivity for $\mathrm{S} 100$ protein in cells located at the periphery of cell nests. Tissue samples from our case were immunoreactive for pan-cytokeratin, Synaptophysin and Chromogranin A (Figure 6, Figure 7 and Figure 8$)$. The Ki-67 proliferative index was $99 \%$ (Figure 9).

SNUC generally is Synaptophysin and Chromogranin negative ${ }^{4,5}$ and ENB is rarely positive for cytokeratin ${ }^{6}$. ENB may be positive for cytokeratin only in the Homer Wright pseudorosette areas ${ }^{4}$. S100, LCA and CD99 stains were all negative in our case, thereby ruling out malignant melanoma, lymphoma and small round blue cell tumors ${ }^{5}$.

Since SCNEC of the nasopharynx is rare, no specific therapeutic guidelines are available. However, therapy is based on evidence regarding the treatment of pulmonary small cell carcinoma ${ }^{3}$. A multimodality treatment has been recommended. It consists of the combination of chemotherapy (cisplatin based) and radiotherapy, with or without surgery $^{8}$. Unfortunately, in SCNEC it is common to find distant metastasis due to notable haematogenous dissemination to lymph nodes, liver, lungs and bone ${ }^{3}$ with rates of metastasis as high as $35 \%^{2,9}$. Our patient was found to have liver metastasis when she was diagnosed with recurrence of her disease. Primary nasopharyngeal neuroendocrine carcinoma with liver metastasis carries a poor prognosis and an overall survival of 10 months ${ }^{12}$. 


\section{CONCLUSIONS}

Head and neck cancer patients are at an elevated risk of developing a second primary tumor in the upper aerodigestive tract attributable to the field cancerization phenomenon ${ }^{1}$. Diagnosis of neuroendocrine carcinoma of the sinonasal tract is a challenge by itself. Ancillary studies like immunohistochemistry play a crucial role in obtaining its diagnosis ${ }^{5}$. Despite the lack of a consensual therapeutic protocol, the multimodality multidisciplinary team approach is needed to treat SCNEC of the nasopharynx ${ }^{3}$. Primary SCNEC of the head and neck region carries a poor prognosis with a median overall survival reported at 14.5 months, and a three-year survival rate of $23.7 \%$.

Conflict of interest: The author and all co-authors have no conflict of interest.

Contribution of authors: All authors have equally contributed to this work.

Acknowledgements: This case report was written by Dr.Madhusudhan Krishnamoorthy. All co-authors have read and agreed to the final version of this manuscript, and have equally contributed to its content, as well as the management of the case.

\section{REFERENCES}

1. Chen CL, Hsu MM. Second primary epithelial malignancy of nasopharynx and nasal cavity after successful curative radiation therapy of nasopharyngeal carcinoma. Hum Pathol. 2000;31 (2):227-32.

2. Bellahammou K, Lakhdissi A, Akkar O, Kouhen F, Rais F, Dahraoui $\mathrm{S}$, et al. Small-cell neuroendocrine carcinoma of nasopharynx: a case report. IJSM. 2017;3(2):132-5.
3. Aguiar A, Jacome M, Azevedo I, Monteiro E, Castro V. Small-cell neuroendocrine carcinoma originating from the nasopharynx: report of a rare case. Rep Radiother Oncol. 2015;2(3):e3814. DOI: $10.17795 /$ rro-3814.

4. Nagy AA, Trombitas V, Vlad D, Albu S. Sinonasal neuroendocrine carcinoma - a case report. Romanian Journal of Rhinology. 2014;4(14):117-20.

5. Radhakrishnan D, Supriya NK, Varma KR. Sinonasal neuroendocrine carcinoma in a young female - a case report. Journal of Clinical and Diagnostic Research. 2017;11(11):ED05-ED06. Available from: http:/ /www.jcdr.net/article_fulltext.asp?issn=0973-709x\&year=2017 \&month=November\&volume $=11$ \&issue $=11$ \&page $=$ ED05\&id=10907 .

6. Montone KT. The differential diagnosis of sinonasal/nasopharyngeal neuroendocrine/neuroectodermally derived tumors. Arch Pathol Lab Med. 2015;139(12):1498-507. DOI: 10.5858/arpa.20140383-RA.

7. Azevedo D, Rios E, Vendeira L, Sarmento C. Small cell neuroendocrine carcinoma of the nasopharynx: a rare case report. Autops Case Rep. 2017;7(1):31-5. DOI: 10.4322/acr.2017.002.

8. Lin CH, Chiang TP, Shum WY, Hsu CH, Tsai YC, Tsao TY, et al. Primary small cell neuroendocrine carcinoma of the nasal cavity after successful curative therapy of nasopharyngeal carcinoma: a case report. Kaohsiung J Med Sci. 2009;25(3):145-50. https://doi. org/10.1016/S1607-551X(09)70054-3.

9. Shah K, Perez-Ordóñez B. Neuroendocrine neoplasms of the sinonasal tract: neuroendocrine carcinomas and olfactory neuroblastoma. Head Neck Pathol. 2016;10(1):85-94. DOI: 10.1007/ s12105-016-0696-7. Epub 2016 Feb 1.

10. Silva EG, Butler JJ, MacKay B, Goepfert H. Neuroblastomas and neuroendocrine carcinomas of the nasal cavity. A proposed new classification. Cancer. 1982;50(11):2388-405.

11. Rosenthal DI, Barker JL, El-Naggar AK, Glisson BS, Kies MS, Diaz EM, et al. Sinonasal malignancies with neuroendocrine differentiation: patterns of failure according to histologic phenotype. Cancer. 2004;101(11):2567-73.

12. Guo C, Pan Q, Su M, Li R. Clinical immunophenotype of nasopharyngeal neuroendocrine carcinoma with metastatic liver cancer. Clin Chim Acta. 2017;471:283-5. DOI: 10.1016/j.cca.2017.06.016. Epub 2017 Jun 17. 